ТЕЛЕГИН А. Н., САРИЛОВ М. Ю.

ПОВЫШЕНИЕ ЭФФЕКТИВНОСТИ МАССООБМЕННЫХ АППАРАТОВ ЗА СЧЕТ ОБОСНОВАННОГО ВЫБОРА КОНТАКТНЫХ УСТРОЙСТВ

Телегин А. Н., Сарилов М. Ю.

A. N. Telegin, M. Yu. Sarilov

ПОВЫШЕНИЕ ЭФФЕКТИВНОСТИ МАССООБМЕННЫХ АППАРАТОВ ЗА СЧЕТ ОБОСНОВАННОГО ВЫБОРА КОНТАКТНЫХ УСТРОЙСТВ

\title{
THE EFFICIENCY INCREASE OF MASS EXCHANGE APPARATUS WITH THE HELP OF CONTACT DEVICES
}

Телегин Андрей Николаевич - магистрант кафедры «Машины и аппараты химических технологий» Комсомольского-на-Амуре государственного технического университета (Россия, г. Комсомольск-наAмype). E-mail: ship.1994@mail.ru.

Mr. Andrey N. Telegin - Master's Degree student, Chemical Industry Machinery and Equipment Department, Komsomolsk-on-Amur State Technical University (Russia, Komsomolsk-on-Amur). E-mail: ship.1994@mail.ru.

Сарилов Михаил Юрьевич - доктор технических наук, профессор кафедры «Машины и аппараты химических производств» Комсомольского-на-Амуре государственного технического университета (Россия, г. Комсомольск-на-Амуре). E-mail: sarilov@knastu.ru.

Mr. Mikhail Yu. Sarilov - D.Sc. in Engineering, Head of the Chemical Industry Machinery and Equipment Department, Komsomolsk-on-Amur State Technical University (Russia, Komsomolsk-on-Amur). E-mail: sarilov@knastu.ru.

Аннотация. В процессе выбора контактного устройства в массообмене обычно используется устройство, которое позволяет повысить выработку определенного продукта. Рассмотрим на примере получения продуктов нефтегазового комплекса. Во время проектирования колонн тарельчатого типа для проведения массообменных процессов в нефте- и газоперерабатывающей промышленности особое внимание уделяется выбору контактных элементов. Оптимальный подбор конструктивных параметров тарелки и направления движения потоков дает возможность создать такие гидродинамические условия проведения процесса массообмена, при которых достигается минимальное значение брызговынесения, равномерное распределение жидкости между тарелками, эффективное контактирование сплошной и дисперсной фаз.

Summary. In the process of selecting a contact mass exchange apparatus the device which can increase the production capacity is commonly used. The authors consider the example of producing oil and gas products. During the designing of plate-type columns for mass exchange processes in the oil and gas processing industry the selection of the contact elements is focused on. Optimum selection of the design parameters of the plates and the flow streamline makes it possible to create such hydrodynamic conditions of the mass exchange process, in which the minimum value of splashing removal is achieved, as well as even distribution of liquid between the plates, effective contacting of the continuous and dispersed phases.

Ключевые слова: массообмен, контактные устройства, аппарат, выбор, эффективность.

Key words: mass exchange, contact device, an apparatus, selection, efficiency.

УДК 650.98

\section{Введение}

Тенденция использования вихревых контактных устройств получила широкое внедрение в процессах абсорбции и ректификации [1]. В исследованиях в основном оценивается влияние способов создания закрученного газового потока на гидродинамику контактных ступеней и почти не уделяется внимание созданию новой организации движения потоков в пределах тарелки и в колонном аппарате [4]. Кроме того, недостаточно изучается вопрос влияния конструкции контактной ступени на эффективность сепарации брызг жидкости [6]. 
Целью написания статьи является обоснование возможности создания противоточного движения потоков на тарельчатых массообменно-разделительных контактных устройствах и разработка конструкции тарелки с новой организацией движения контактирующих потоков. Противотечное движение в противовес перекрестному [2] делает возможным равномерное распределение движущей силы на каждом из контактных устройств колонны [7]. К преимуществам предложенной схемы движения фаз относятся низкое гидравлическое сопротивление [5] и возможность работы колонны со значительными затратами в газовой и жидкой фазах [3].

Основные задачи работы:

- проведение экспериментальных исследований гидродинамики движения потоков в пределах массообменно-сепарационной секции с прямоточно-центробежными элементами, определение режимов работы тарелки и ее гидравлического сопротивления;

- компьютерное моделирование движения газового потока в пределах прямоточноцентробежного элемента, определение его основных геометрических характеристик;

- сопоставление результатов эксперимента и компьютерного моделирования с целью определения оптимальных условий создания противоточного движения потоков на тарелке;

- корректировка и дополнение методики гидродинамического расчета контактной ступени и конструктивного расчета колонны по результатам проведенных исследований;

- поиск оптимальных размеров контактной ступени в зависимости от нагрузок по сплошной и дисперсной фазам.

В ходе проведения экспериментальных исследований массообменно-сепарационной контактной ступени выделено четыре гидродинамических режима, которые отличаются степенью завихрения жидкости в прямоточно-центробежном элементе, характером движения жидкости в прямоточно-центробежном элементе, поведением жидкости на полотне контактной ступени и интенсивностью брызговынесения с контактной ступени.

При соотношении жидкости к газу $\mathrm{L} / \mathrm{G}=0,6$ - 0,7 кг/кг объем жидкости, которая поступает к центральной части массообменного элемента полотна тарелки через трубку, частично диспергируется потоком газа (поскольку расход газа незначителен), а основной объем жидкости сливается по внутренней стенке контактной трубки массообменного элемента. Поток газа, в свою очередь, проходит через центральную часть контактной трубки массообменного элемента. Для данного режима характерно образование единичных пузырьков газа большого размера в слое жидкости; площадь соприкосновения фаз не развита; происходит неравномерный слив жидкости на расположенную ниже тарелку (см. рис. 1, $a$, режим 1).

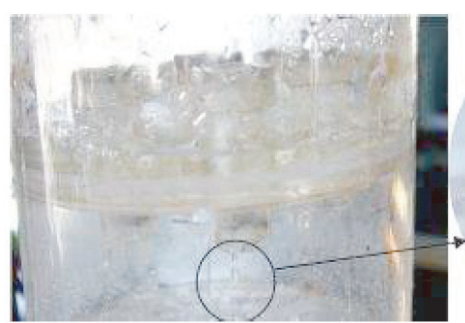

a)

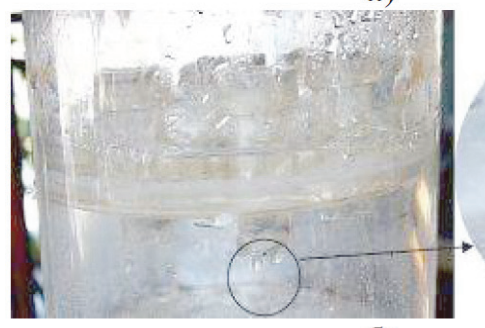

б)
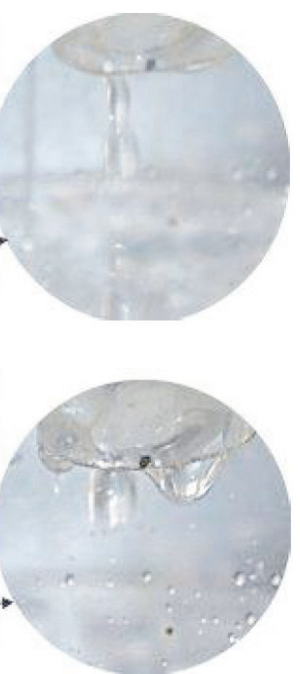

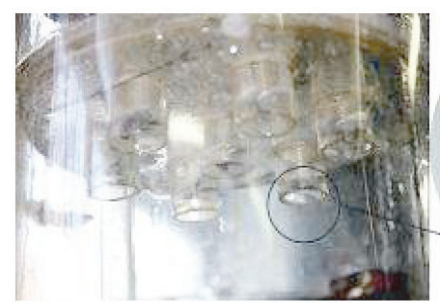

B)

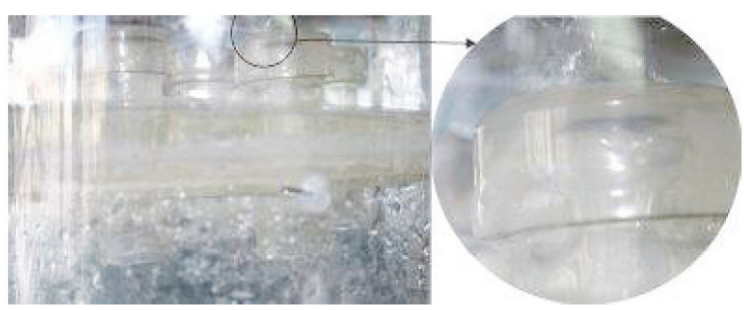

г)

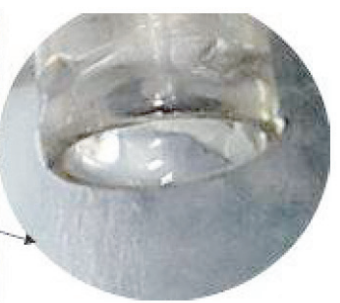

нно-разделительных контактных устройств при соотношении жидкости к газу $\mathrm{L} / \mathrm{G}$, кг/кг: $\mathrm{a}-\mathrm{L} / \mathrm{G}=0,6-0,7 ; \sigma-\mathrm{L} / \mathrm{G}=1,0-1,1 ;$ в $-\mathrm{L} / \mathrm{G}=1,2-1,3 ; \Gamma-\mathrm{L} / \mathrm{G}=1,4-1,5$ 
ТЕЛЕГИН А. Н., САРИЛов М. Ю.

ПОВЫШЕНИЕ ЭФФЕКТИВНОСТИ МАССООБМЕННЫХ АППАРАТОВ ЗА СЧЕТ ОБОСНОВАННОГО ВЫБОРА КОНТАКТНЫХ УСТРОЙСТВ

При существенном повышении соотношения жидкости к газу $\mathrm{L} / \mathrm{G}=1,0-1,1$ кг/кг объем жидкости, которая поступает к центральной части массообменного элемента, в значительной по сравнению с предыдущим режимом мере диспергируется потоком газа, но жидкость еще частично сливается по внутренней стенке контактной трубки массообменного элемента в виде капель. Поток газа, в свою очередь, проходит сквозь слой жидкости с более интенсивным по сравнению с предыдущим режимом образованием вихрей, пузырьков газа и пены; площадь соприкосновения фаз более развита, чем в предыдущем режиме. При этом жидкость отдельными каплями стекает по внутренней стенке патрубка, а остальные жидкости сливаются через пленкосъемник (см. рис. 1, б, режим 2).

Гидродинамический режим с соотношением жидкости к газу $\mathrm{L} / \mathrm{G}=1,2-1,3$ кг/кг характеризуется тем, что объем жидкости, которая поступает к центральной части массообменного элемента полотна тарелки через трубку, полностью диспергируется потоком газа, слив жидкости по внутренней стенке контактной трубки массообменного элемента отсутствует. Поток газа проходит сквозь слой жидкости с интенсивным вихреобразованием и образованием мелкодисперсных пузырьков газа и пены. Таким образом, площадь соприкосновения фаз наиболее развита. При этом жидкость стекает в виде пленки с пленкосъемника, а слив жидкости по внутренней стенке массообменного патрубка отсутствует. Этот режим является оптимальным (см. рис. 1, в, режим 3).

Дальнейшее увеличение соотношения жидкости к газу $(\mathrm{L} / \mathrm{G}=1,4-1,5$ кг/кг) приводит к тому, что объем жидкости, которая поступает к центральной части массообменного элемента полотна тарелки через трубку, диспергируется в меньшей степени, чем при оптимальном режиме, поскольку газ проходит через слой жидкости в виде струй. Пленка жидкости, которая отбрасывается центробежными силами ко внутренней стенке контактной трубки массообменного элемента в результате завихрения потока, захватывается потоком газа и частично попадает в пленкосъемник вместе с газовым потоком, но большая часть пленки движется с потоком газа на вышерасположенную тарелку сквозь центральное отверстие верхней контактной трубки массообменного элемента, не попадая в пленкосъемник, то есть для данного режима характерно повышенное брызговынесение и значительное уменьшение площади соприкосновения фаз (см. рис. 1, 2, режим 4).

При расчетах гидравлического сопротивления сухих тарелок следует руководствоваться полученными экспериментально значениями коэффициента сопротивления массообменносепарационных устройств $\xi=3,5-6$ (см. рис. 2).

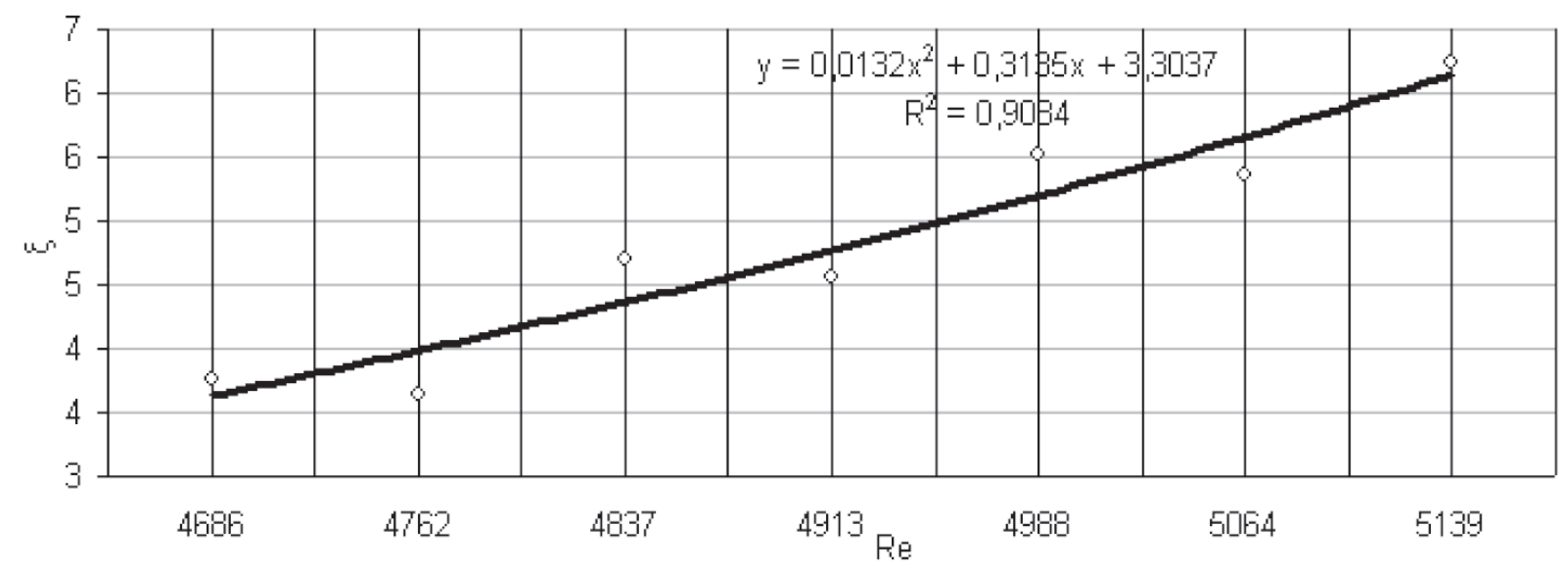

Рис. 2. Зависимость коэффициента сопротивления сухих тарелок от значения критерия Рейнольдса

Результаты, иллюстрирующие зависимость гидравлического сопротивления контактного устройства в каждом из исследуемых гидродинамических режимов, приведены на рис. 3. 


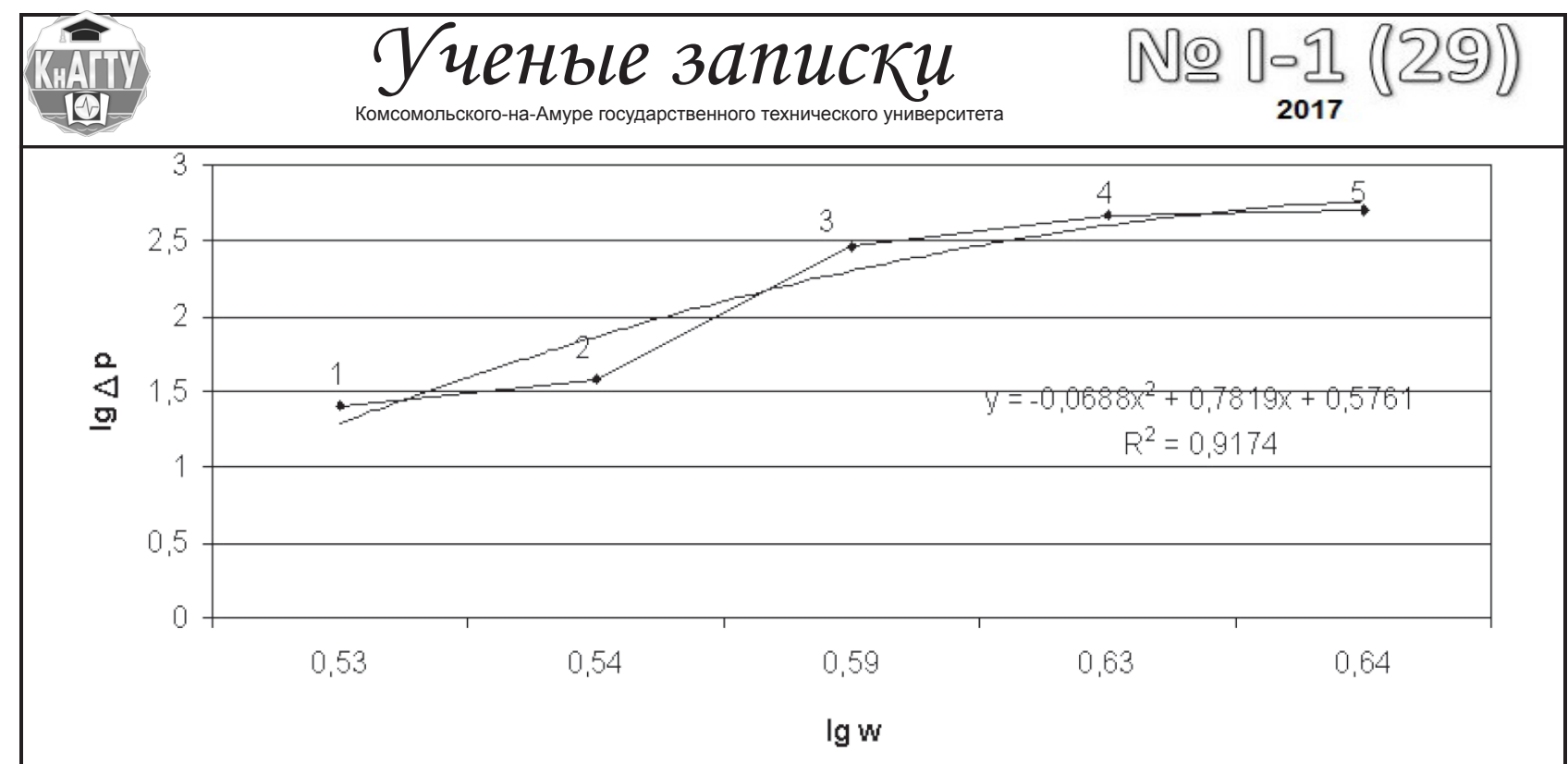

Рис. 3. Логарифмическая зависимость гидравлического сопротивления контактного устройства от скорости газа: $1-2$ - режим 1;2-3 - режим 2 ; $3-4$ - режим $3 ; 4-5$ - режим 4

Зависимость гидравлического сопротивления контактного устройства от соотношения расходов дисперсной (жидкость) и сплошной (газ) фаз показано на рис. 4. Диапазон устойчивой работы тарелки - второй режим.

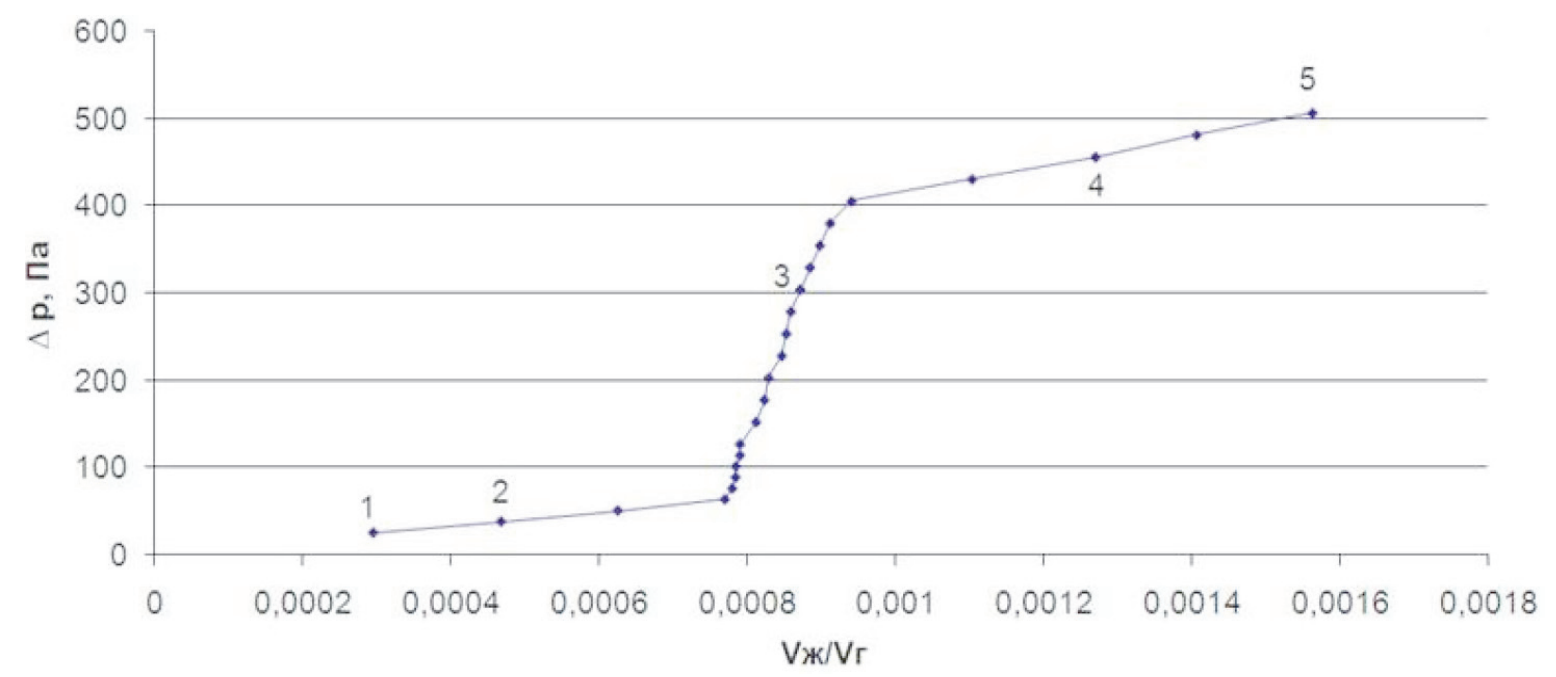

Рис. 4. Зависимость гидравлического сопротивления контактного устройства от соотношения объемных расходов жидкости и газа: $1-2$ - режим 1;2-3 - режим 2; 3-4 - режим 3; 4-5 - режим 4

Рассмотреть условия создания противоточного движения фаз на отдельных контактных ступенях, а также основные гидродинамические закономерности движения высокотурбулентных потоков в прямоточно-центробежных элементах массообменно-сепарационных тарелок стало возможным способом математического (компьютерного) моделирования в среде программного продукта SolidWorks Simulation (http:/www.solidworks.com). В процессе моделирования были испытаны различные варианты процесса с изменением конструктивных параметров контактного массообменно-сепарационного элемента. Смоделированные трехмерные модели течений газового потока с высокодисперсной капельной жидкостью по элементам массообменно-сепарационных устройств визуализированы методами компьютерной графики (см. рис. 5). 

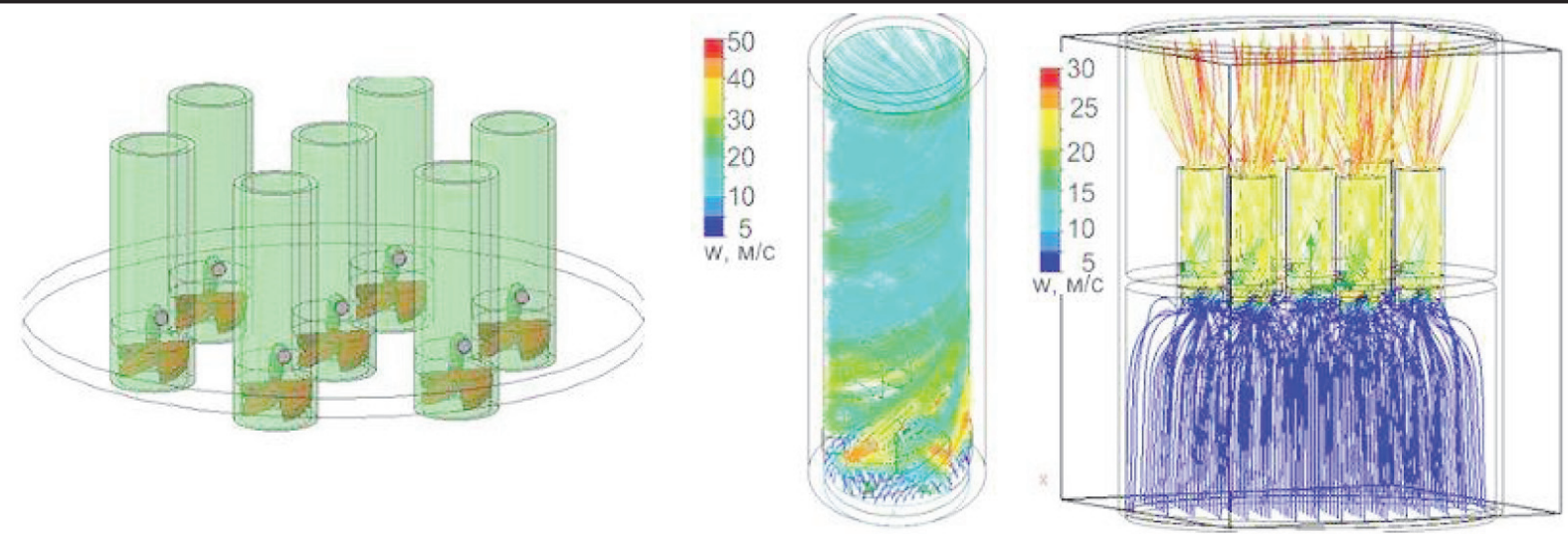

Рис. 5. 3D-модель тарелки с контактными элементами и траектории движения газового потока с заливками по значению локальных скоростей

\section{Выводы}

На основании теоретического анализа полученных результатов физических (экспериментальных) и математических (компьютерных) моделирований предложена новая полезная модель контактного устройства (тарелки) с массообменно-сепарационными элементами для колонных абсорберов осушки природного газа.

Внесены изменения в конструкции прямоточно-центробежного элемента (направленное стекание жидкости через пленкосъемник и сегментные отверстия в полотне тарелки), создаются выгодные условия для противоточного движения фаз на контактных тарелках и в пределах колонного аппарата.

Исследования гидродинамики таких аппаратов показали, что использование тарелок с противоточным движением фаз на контактных ступенях и прямоточным в центробежных массообменно-сепарационных элементах позволяет значительно увеличить поверхность контакта фаз и уменьшить брызговынесение. Внедрение противоточного движения в противовес перекрестному делает возможным более равномерное распределение и одновременное увеличение движущей силы на каждом из контактных устройств колонны. К преимуществам предложенной схемы движения фаз относится низкое гидравлическое сопротивление из-за условий работы колонны при значительных затратах по газовой и жидкой фазам.

\section{ЛИТЕРАТУРА}

1. Антонов, М. А. Компьютерное моделирование жесткости несущих элементов контактных устройств массообменного аппарата / М. А. Антонов, Р. З. Латфуллин, И. А. Сабанаев // Вестник Казанского технологического университета. - 2016. - Т. 19. - № 23. - С. 82-84.

2. Массообмен в насадочной колонне экстрактивной ректификации / И. В. Иванов [и др.] // Теоретические основы химической технологии. - 2016. - Т. 50. - № 5. - С. 485-495.

3. Кологривов, М. М. Численное моделирование тепло- и массообмена в эжекционном аппарате М. М. Кологривов, В. П. Бузовский // Инженерно-физический журнал. - 2016. - Т. 89. - № 1. - С. 158-169. 4. Лапшина, С. В. Оценка прочностных характеристик оборудования / С. В. Лапшина, Е. М. Тупикина // В книге: V Международная конференция-школа по химической технологии XT'16: сборник тезисов докладов сателлитной конференции XX Менделеевского съезда по общей и прикладной химии. - 2016. - С. 89-90.

5. Лымбина, Л. Е. Особенности тепло- и массообменных процессов в технических водоемах // Энерго- и ресурсосбережение в теплоэнергетике и социальной сфере: материалы Междунар. науч.-тех. конф. студентов, аспирантов, ученых. - 2016. - Т. 4. - № 1. - С. 160-163.

6. Фарахов, Т. М. Обобщенные гидравлические и массообменные характеристики новых контактных насадок колонных аппаратов / Т. М. Фарахов, М. М. Фарахов, Е. А. Лаптева // Химическая промышленность сегодня. - 2016. - № 2. - С. 50-56.

7. Фарахов, М. М. Конструирование высокоэффективных массообменных устройств газожидкостного контакта / М. М. Фарахов, Е. А. Лаптева // Современные тенденции развития науки и технологий. - 2016. № 7-2. - C. 72-77. 